\title{
Development of a precision multimodal surgical navigation system for lung robotic segmentectomy
}

\author{
Jean Marc Baste ${ }^{1}$, Valentin Soldea ${ }^{1,2}$, Samy Lachkar ${ }^{3}$, Philippe Rinieri ${ }^{1}$, Mathieu Sarsam ${ }^{1}$, Benjamin \\ Bottet $^{1}$, Christophe Peillon ${ }^{1}$
}

${ }^{1}$ Department of General and Thoracic Surgery, Rouen University Hospital, Rouen, France; ${ }^{2}$ Department of Pathology, University of Medicine and Pharmacy, Targu Mures, Roumania; ${ }^{3}$ Department of Pulmonology and CIC-CRB 1404, Rouen University Hospital, Rouen, France

Correspondence to: Jean Marc Baste, MD, PhD. Department of Thoracic Surgery, Rouen University Hospital Charles Nicolle, 1 rue de Germont, 76031 Rouen, France. Email: jean-marc.baste@chu-rouen.fr.

\begin{abstract}
Minimally invasive sublobar anatomical resection is becoming more and more popular to manage early lung lesions. Robotic-assisted thoracic surgery (RATS) is unique in comparison with other minimally invasive techniques. Indeed, RATS is able to better integrate multiple streams of information including advanced imaging techniques, in an immersive experience at the level of the robotic console. Our aim was to describe three-dimensional (3D) imaging throughout the surgical procedure from preoperative planning to intraoperative assistance and complementary investigations such as radial endobronchial ultrasound (R-EBUS) and virtual bronchoscopy for pleural dye marking. All cases were operated using the DaVinci System $^{\mathrm{TM}}$. Modelisation was provided by Visible Patient ${ }^{\mathrm{TM}}$ (Strasbourg, France). Image integration in the operative field was achieved using the Tile Pro multi display input of the DaVinci console. Our experience was based on 114 robotic segmentectomies performed between January 2012 and October 2017. The clinical value of 3D imaging integration was evaluated in 2014 in a pilot study. Progressively, we have reached the conclusion that the use of such an anatomic model improves the safety and reliability of procedures. The multimodal system including 3D imaging has been used in more than 40 patients so far and demonstrated a perfect operative anatomic accuracy. Currently, we are developing an original virtual reality experience by exploring 3D imaging models at the robotic console level. The act of operating is being transformed and the surgeon now oversees a complex system that improves decision making.
\end{abstract}

Keywords: Lung segmentectomy; robotic surgery; image assisted surgery; augmented reality; surgical navigation/ operative planning

Submitted Dec 21, 2017. Accepted for publication Jan 03, 2018.

doi: $10.21037 /$ jtd.2018.01.32

View this article at: http://dx.doi.org/10.21037/jtd.2018.01.32

\section{Introduction}

The use of sub lobar anatomical resection is likely to increase, as confirmed by ongoing clinical trials for the management of very early stage lung carcinoma (1). Anatomical variation has rarely been described unless for specific lobes, for example the middle and lower lobe (2). However, from a technical point of view segmentectomy is a difficult procedure to perform and is reserved for highly experienced teams especially when a minimally invasive approach is proposed $(3,4)$.
We have identified four main technical difficulties in performing successful minimally invasive segmentectomy.

* Constant anatomical variation of the bronchovascular elements and irregular/unpredictable spatial conformation of a given pulmonary segment;

* Unclear delimitation of the intersegmental plan;

* Difficult assessment of a safe resection margin;

* Lymphadenectomy of the intrapulmonary nodes.

Our aim was to describe three-dimensional (3D) imaging throughout the surgical procedure from preoperative planning to intraoperative assistance and complementary 
Inception of our Multimodal imaging system for robotic segmentectomy

Our Robotic program has started in 2012 and we have performed Robotic segmenetctomy since 2013 (5). In 2017 segmentectomy are performed > $90 \%$ by RATS. The robotic platform associated with this multi-modal imaging system should overcome the different surgical challenges represented by (I) constant anatomical variations (shape of segment, artery, brocnhus, vein), (II) the intersegmental plan, (III) the location of the tumor and the intersegmental plan, (IV) lymphadenectomy of the area $11,12$.

3D reconstruction (Visible Patient ${ }^{\mathrm{TM}}$ ) for pre-
operative planning Pilot Study (6)
2013-2015
2013-2015

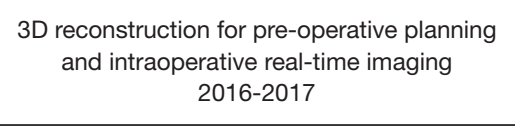
2016-2017
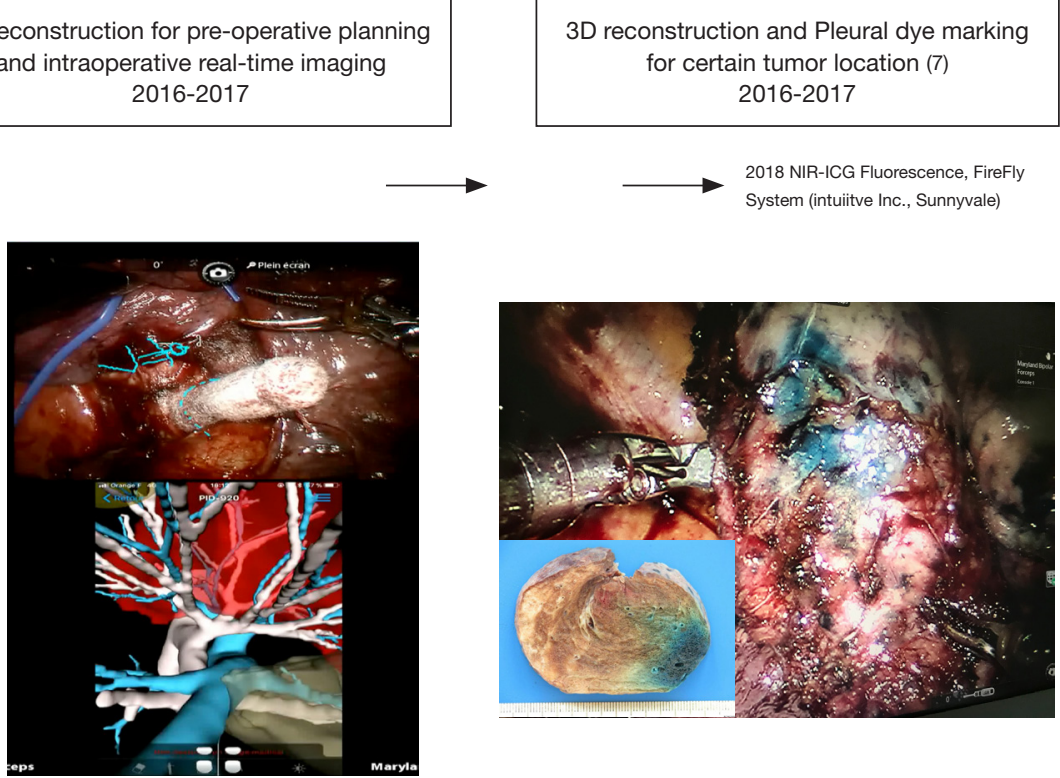

During dissection, the 3D model injected in the operative field (virtual reality) help us to check operative field (virtual reality) help us to check
artery, bronchus, vein and segmental border before stapling. Left S2 artery and bronchus control.
$3 \mathrm{D}$ reconstruction showing the tumor location in the good segment and the shape of it. Great help to choose the adequate segment and for imaging the intersegmental plan. Iphone support or touchless screen.

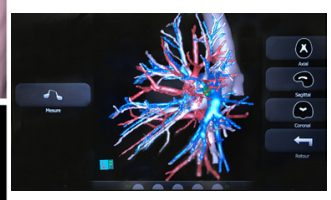

Figure 1 Flow chart of the implementation of our robotic technique of segmentectomy reinforced by our multimodal imaging system (5-7).

Table 1 Procedures performed using our multimodal system

\begin{tabular}{lccc}
\hline Years & $\begin{array}{c}\text { 3D model operative } \\
\text { planning }\end{array}$ & $\begin{array}{c}\text { 3D model operative planning and virtual } \\
\text { reality }\end{array}$ & $\begin{array}{c}\text { Pleural dye marking associated with } \\
\text { 3D model }\end{array}$ \\
\hline $2014-2016$ & 15 & 5 & 0 \\
$2016-2017$ & 40 & 40 & 15 \\
Operative anatomic accuracy & $100 \%$ & $100 \%$ & $100 \%$ \\
\hline
\end{tabular}

3D, three-dimensional.

invasive investigations such as radial endobronchial ultrasound (R-EBUS) and pleural dye marking. This work emphasizes the development of the concept of imageassisted surgery: a step by step evolution (Figure 1).

\section{Experience}

Our experience was based on 114 robotic segmentectomies performed between January 2012 and October 2017.
All indications for the proposed segmentectomy were in accordance with preoperative multidisciplinary meeting decisions ( $<2 \mathrm{~cm}$ proven or suspected lung cancer or pulmonary metastasis).

The technique of our robotic segmentectomy (a simplified 3-arm approach) has been described previously (5). The clinical value of the $3 \mathrm{D}$ model was validated in 2014 in a pilot study (6). Progressively, we have reached the conclusion that the availability of such 
an anatomic model demonstrated a perfect operative anatomic accuracy with regard to the bronchial and vessels distribution, and improved the safety and reliability of procedures. Our point of view regarding the benefits of using $3 \mathrm{D}$ reconstruction in surgical practice is shared by other teams (8). We have used this complete protocol in routine practice for more than one year (Table 1), (Figure 2).

\section{Description of image acquisition and workflow}

The rendering of the $3 \mathrm{D}$ models is outsourced to a private

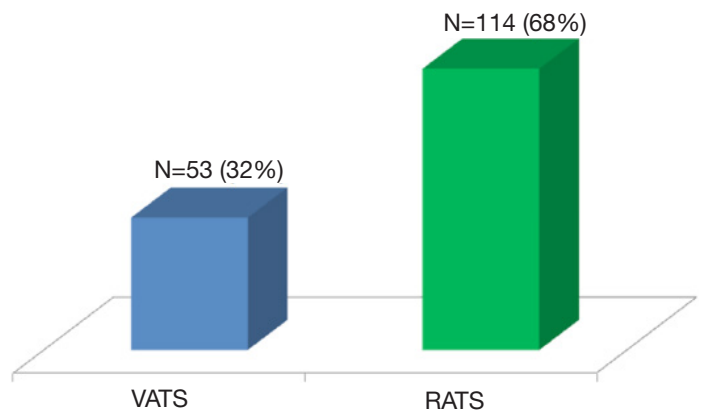

Figure 2 Minimal invasive segmentectomies from 2008 to 2017 in our institution epithor registry. VATS, video-assisted thoracic surgery; RATS, robotic-assisted thoracic surgery. company, Visible Patient ${ }^{\mathrm{TM}}$ (Strasbourg, France) (6). This requires no extra work for the referent surgeon. This point is relevant because the $3 \mathrm{D}$ model used for surgery may be complex to build and time consuming. To protect the identity of the patient, data are anonymized. A CT scan is performed by the radiology department. Images (DICOM format) stored in the hospital picture archiving and communication system (PACS) are uploaded to the Therapixel $^{\mathrm{TM}}$ server. Reconstructions are rendered by specialized radiological technicians (Visible Patient ${ }^{\mathrm{TM}}$ employees) who contour all anatomical structures (arterial, venous and bronchial up to the fifth level of division). Lobar and sublobar segmentation are performed automatically by the software. The completed 3D models are approved for clinical use under the Council of Europe directive CEO459, FDA and in Canada (no 94537). The model itself is portable (6), and its different properties can be explored with dedicated software. The application used for accessing the models_- "Anywhere imaging" provides easy access across multiple hardware platforms (mainstream computers, tablets, smartphones) and permits external image projection. This avoids the use of high performance workstations (Figure 3).

The main functions of the completed $3 \mathrm{D}$ models that can be explored are:

* Individual exploration of the distinct anatomical
1. Outpatient clinic

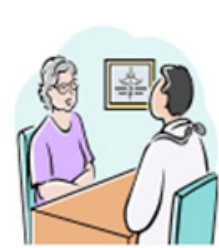

2. Surgeon request a ctscan with injection for $3 D$ reconstruction via web visible patient

$\rightarrow$ Command number anonimised

$\rightarrow 5$ minutes max for the surgeon to pass the command

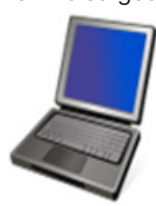

3. AngioCTScan

2 weeks before the surgery

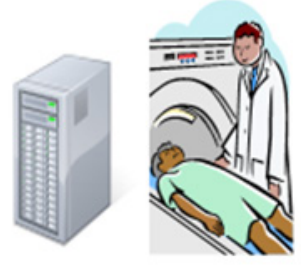

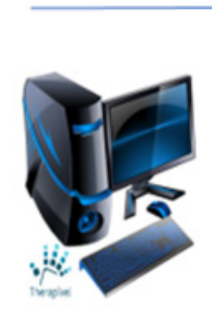

4. Transfer of the 3D model to the visible patient software and anywhere sofware (therapixel)

\section{(1)}

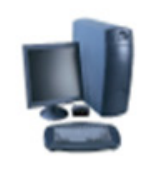

5. Surgeon has access to the 3D model on his tablet or mobile phone via VP app, or anywhere sofware and VP software
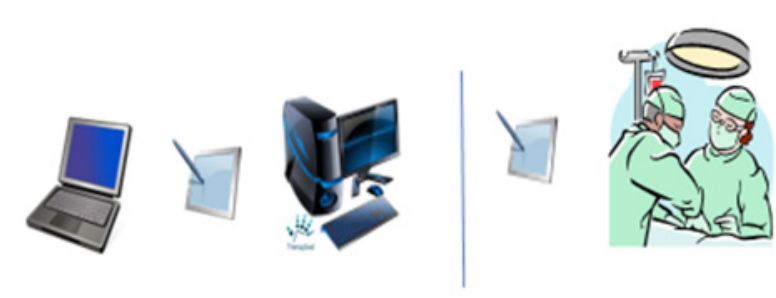

6. Use of the 3D model for the operative planning and surgery assisting

- on tablet (soft VP)

- From therapixel software anywhere

Figure 3 The workflow to get 3D reconstruction from outpatients' clinic to the surgical field. 3D, three-dimensional. 

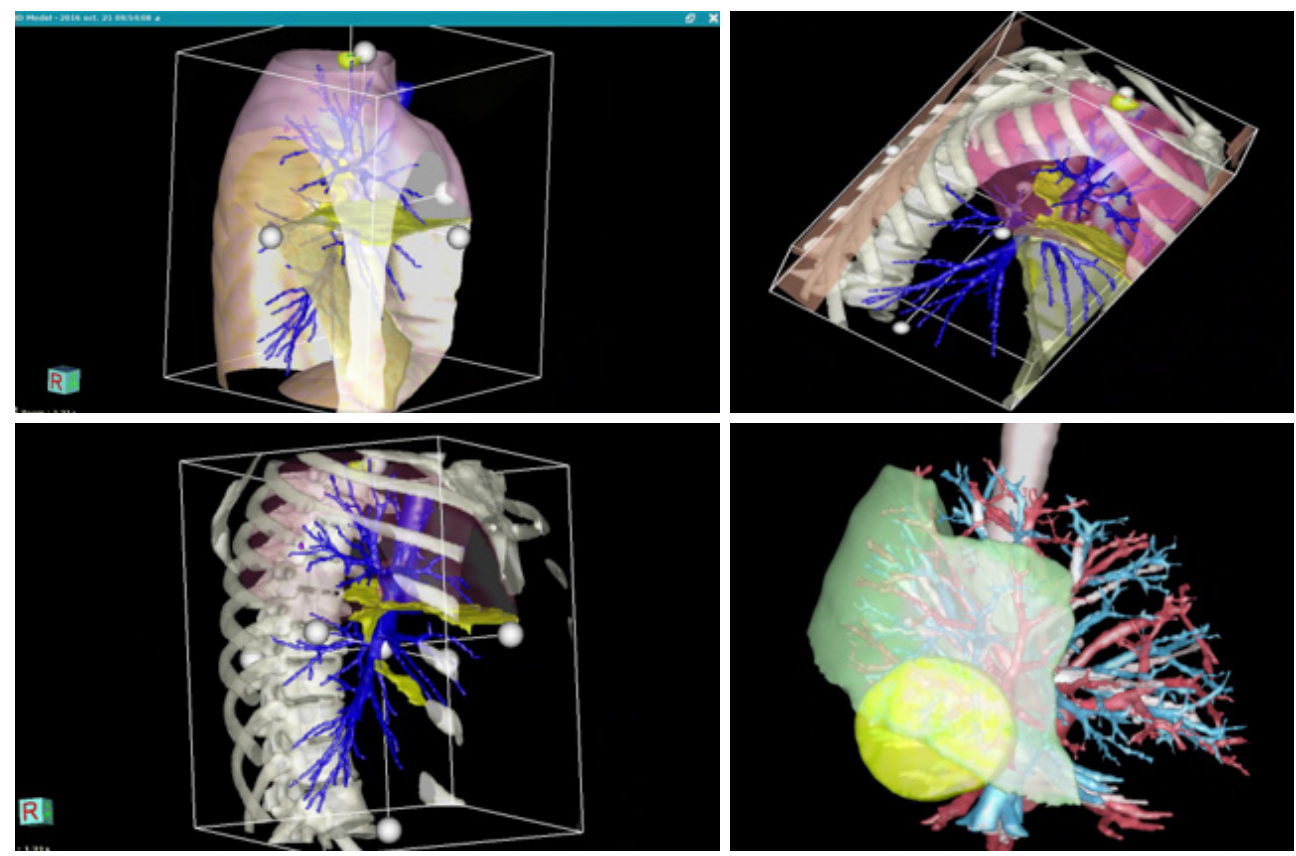

Figure 4 Operative planning using the 3D model provided by VP® using the software Anywhere by Therapixel@. Evolution with segmentation at the end of 2016 (right corner). 3D, three-dimensional.

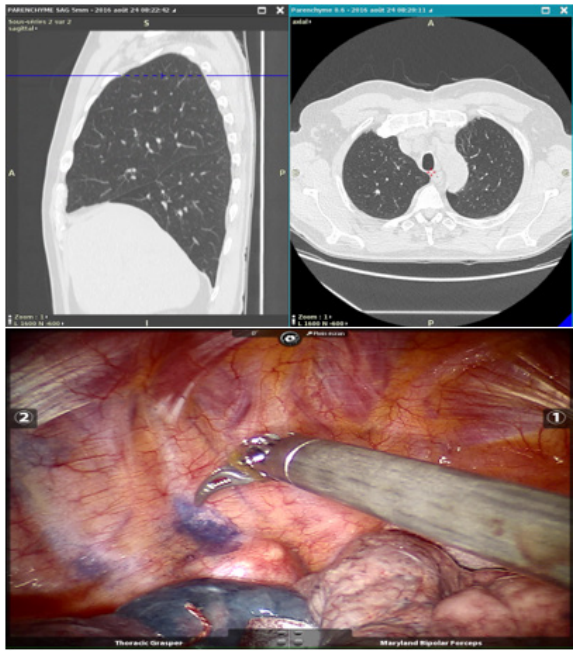

Figure 5 S1 segmentectomy with pleural dye marking with methylene blue without $3 \mathrm{D}$ reconstruction. $3 \mathrm{D}$, three-dimensional.

bronchovascular elements;

* Virtual resections (up to level of the independent segment);

* Volume calculation of each segment-allowing assessment of postoperative volume to analyze the impact on the predictive postoperative respiratory function;

* Simulation of safety margin-important in assessing the feasibility of the proposed resection.

For advanced surgical navigation in the operating room we also use a customized hardware platform-Fluid ${ }^{\mathrm{TM}}$ provided by Therapixel ${ }^{\mathrm{TM}}$ (Valbone, France), (this is rated as a class II medical device) that uses hand gestures for touchless control, allowing the surgeon to remain sterile while exploring the imagery. We also use the same system for video-assisted thoracic surgery (VATS) procedures (Figures 4-7).

Continuing the integration of the $3 \mathrm{D}$ model to a higher level is done by displaying it in the direct field of view of the operating surgeon at the level of the robotic console, through a simple HDMI connection from a portable device (6). The surgeon maintains the option of exploring all the functions of the model. The control of the different visible layers is done manually, by increasing the transparency of the desired anatomical layers. This provides a virtual reality experience for the surgeon. A true fully automated superimposition of the model on the intraoperative view or "augmented reality" will soon be available. However, it is not yet possible because of the physical state of the deflated lung during selective ventilation. 

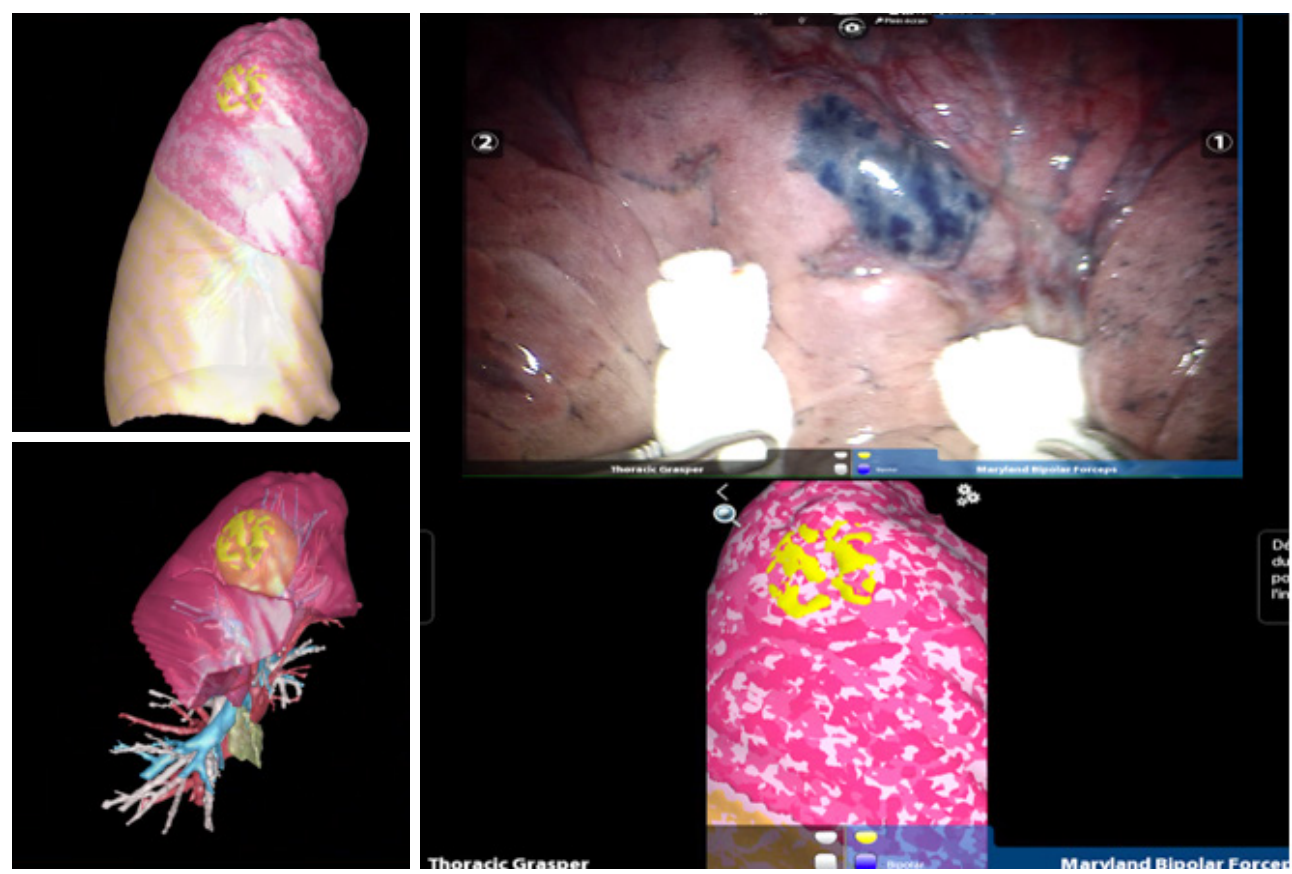

Figure 6 Blue pleural dye marking for lesion close to the intersegmental plan S1/S2.

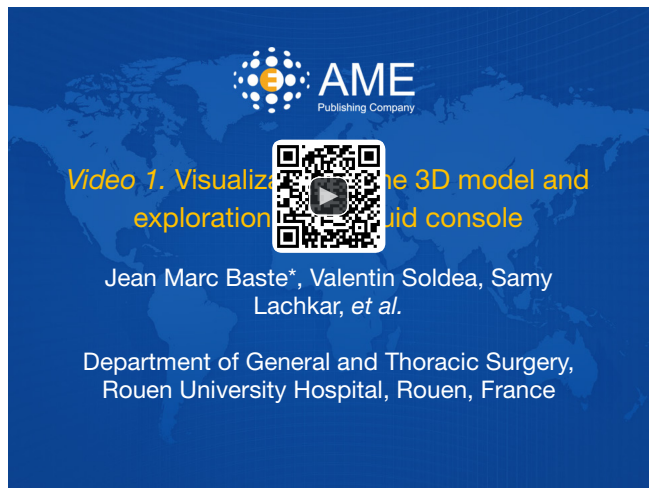

Figure 7 Visualization of the 3D model and exploration on the fluid console (9). 3D, three-dimensional.

Available online: http://www.asvide.com/article/view/24363

\section{Complementary procedures: pleural dye marking}

Several cases $(\mathrm{n}=15)$ were done using pleural dye marking by R-EBUS and virtual bronchoscopy especially when the lesion was close to the intersegmental plan in order to localize small peripheral lung nodules. We added this latest procedure to our $3 \mathrm{D}$ reconstruction technique because previously we had missed one lesion in our series due to the proximity of the intersegmental plan. The efficacy of this procedure has been assessed in a clinical study (7).

We reported in this study that 25 nodules, including 6 ground glass opacities, were resected in 22 patients by video-assisted thoracoscopic wedge resection $(n=11)$ or robotic-assisted thoracoscopic surgery (10 segmentectomies and 1 wedge resection). The median greatest diameter of nodules was $8 \mathrm{~mm}$. No conversion to open thoracotomy was needed. The endoscopic procedure added an average 10 minutes to surgical resection. The dye was visible on the pleural surface in all cases for segmentectomy. Histological diagnosis and free margin resection were obtained in all cases. Median skin to skin operating time was 90 minutes for robotic segmentectomy. The same operative precision was considered impossible by the surgeon without dye marking in the 10 cases of segmentectomy.

The endoscopic procedure is performed without fluoroscopy, under general anesthesia in the operating room immediately before minimally invasive surgery. General endotracheal anesthesia is induced with a single-lumen endotracheal tube in the operating room. The endoscopy is performed using a BF-MP60F endoscope (Olympus ${ }^{\mathrm{TM}}$, Tokyo, Japan) with a 4-mm outer diameter and 2-mm working channel. The ultrasound probe used is a UMS20-17S radial probe (Olympus ${ }^{\mathrm{TM}}$, Tokyo, Japan) with a 1.4-mm diameter and whose distal end is introduced into the 


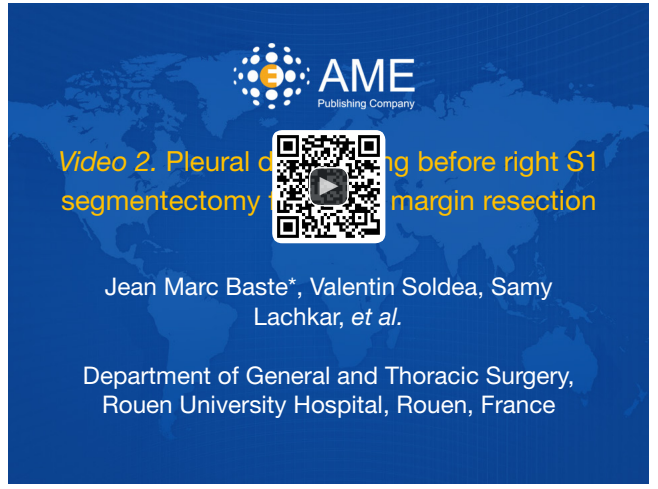

Figure 8 Pleural dye marking before right S1 segmentectomy for better margin resection (10).

Available online: http://www.asvide.com/article/view/24364

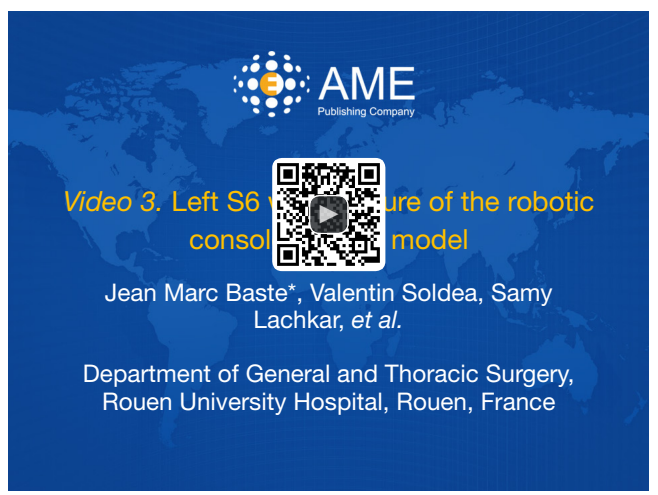

Figure 9 Left S6 with capture of the robotic console and 3D model (11). 3D, three-dimensional.

Available online: http://www.asvide.com/article/view/24365

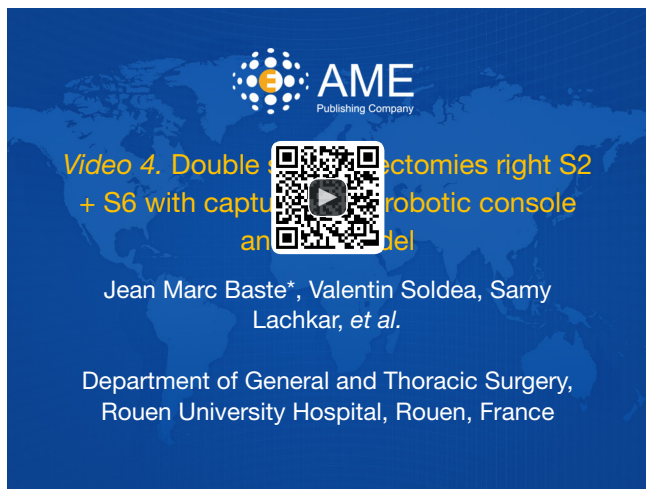

Figure 10 Double segmentectomies right S2 + S6 with capture of the robotic console and 3D model (12). 3D, three-dimensional. Available online: http://www.asvide.com/article/view/24366 1.9-mm diameter guide sheath.

Before each procedure, the nodule location is mapped using virtual bronchoscopy software in order to identify the smallest bronchus leading to the lesion (Lungpoint Planning Bronchus ${ }^{\mathrm{TM}}$, USA). When the fiberscope reaches the most distal sub-segmental bronchus along the route previously defined in virtual bronchoscopy, the guide sheath with the R-EBUS probe is inserted into the working channel. If the lesion is (or not) visualized by ultrasound, the probe is pushed far away into the bronchus in order to be close to the pleural space without fluoroscopy guidance. The probe is removed and the guide sheath left in place within the working channel. Then, $1 \mathrm{~mL}$ of methylene blue is injected in the guide sheath with $20 \mathrm{~mL}$ of air (in order to push the methylene blue dye inside the guide sheath). The bronchoscope is then removed, and the patient is intubated with a double-lumen tube and appropriately positioned (lateral decubitus position) for minimally invasive resection of the lung lesion [VATS or Robotic-assisted thoracic surgery (RATS)]. On inspection of the lung surface, the blue methylene dye is visualized in order to perform a wedge resection or a segmentectomy with endoscopic stapler (Figures 5,8).

\section{Examples of procedures performed using our multimodal protocol}

The first segmentectomy presented here was performed for the resection of a nodular lesion of the left segment 6 , diagnosed on a surveillance CT scan after a renal carcinoma. The lesion was $9 \mathrm{~mm}$ in diameter, and was in the lower part of the segment. With the aid of the 3D images, we quickly identified an anatomical variation with two distinct arteries for the S6. There was no anatomical variation concerning the S6 vein. We used a standardized approach for dissection considering three target areas, in a clockwise direction (pulmonary ligament, posterior pulmonary hilum and fissure. The intervention was uneventful. Biopsy confirmed renal metastasis, all the lymph nodes were negative (Figure 9).

The second example presented was a more complex double segmentectomy right S2 and S6 for two ground glass opacities correlated with a positive pet scan. Here, the model allowed precise identification of the A2 artery and B2 bronchus. The vein for the S2 was stapled with the parenchyma. Dissection and stapling for the S6 was performed with complete dissection of all the broncho- 

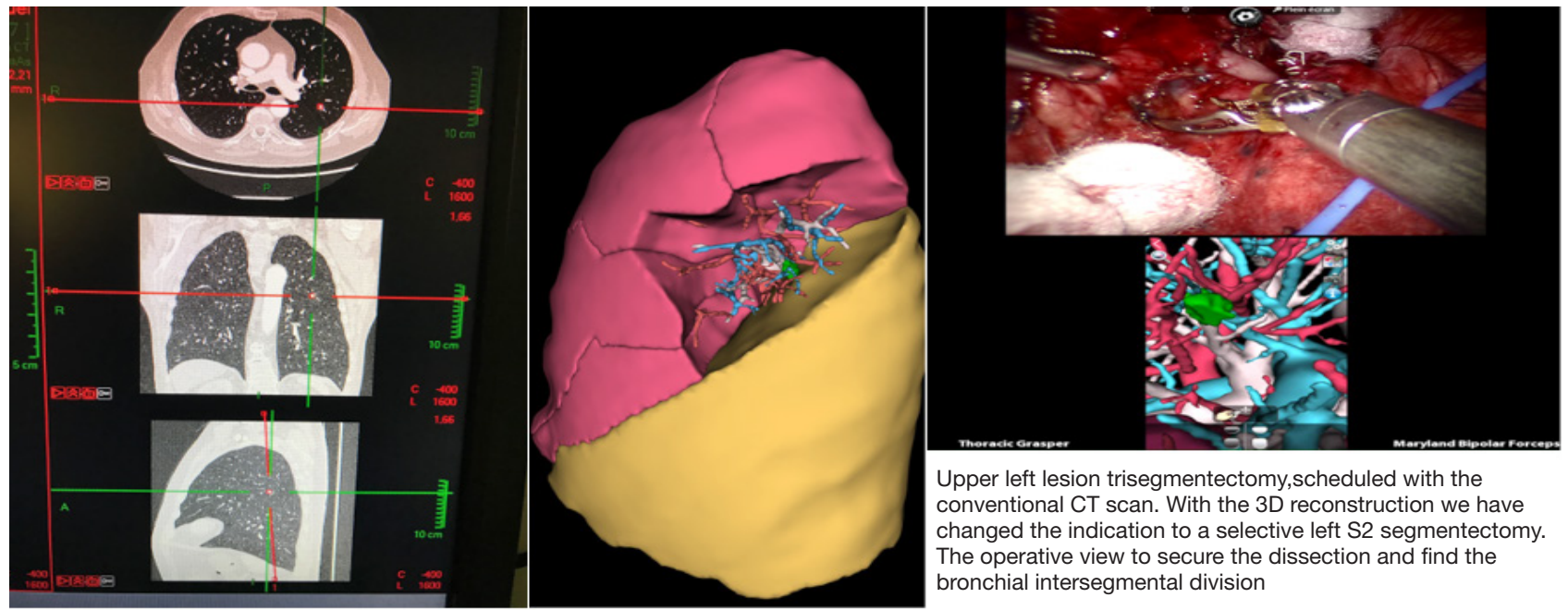

Figure 11 Left S2 segmentectomy.

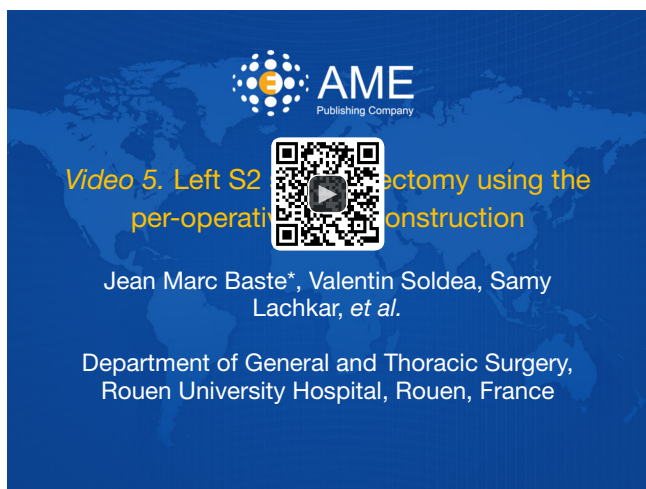

Figure 12 Left S2 segmentectomy using the per-operative 3D reconstruction (13). 3D, three-dimensional.

Available online: http://www.asvide.com/article/view/24367

vascular elements. Biopsy confirmed two distinct adenocarcinoma lesions without positive nodes (Figure 10).

The third example was a highly selective left S2 segmentectomy for a $15-\mathrm{mm}$ nodule. The lesion was difficult to localize on the CT scan, appearing to be located in the posterior part of the lingula. The etiology was unconfirmed, and was accompanied by a positive PET scan. Without the precise localization of the lesion on the preoperative planning this resection would not have been possible. The intervention was uneventful (Figures 11,12).

The fourth example is a planning S6 segmentectomy with a rare anatomical variation with 2 arteries (A6 a,b) and 2 bronchus (B6 a,b) (Figure 13).

\section{Comments}

Lung segmentectomy is gaining increasing interest as both a diagnostic and curative procedure for various small and central lung lesions requiring surgical excision. It is considered oncologically safe and presents the advantage of having a very limited impact on pulmonary function. The technical aspects of the procedure require a thorough understanding of the complex and highly variable pulmonary anatomy which could be improve by 3D model (7).

This multimodal imaging system protocol integrates the preoperative assessment of anatomical conditions and puts them right where they are needed in the operative room. This level of integration is only possible during robotic surgery. This surgical cockpit allows the surgeon to be visually immersed in local conditions. It is a great way to alleviate informational overload. All the mental projections concerning intricate anatomy fit in naturally without supplemental effort from the operator, clearly impacting the decision-making process during critical dissection and no return decisions like stapling of the different bronchovascular elements. We believe that a complete surgical approach using a multimodal imaging system and a robotic platform could prevent intraoperative accidents and postoperative complications, and could improve the general performance of the surgical act.

Such an approach has been described by others using Oisrix $3 \mathrm{D}$ reconstruction and the robotic platform $(7,14,15)$. However, the quality of the Oisrix 3D model was less accurate than the $3 \mathrm{D}$ model provided by Visible 


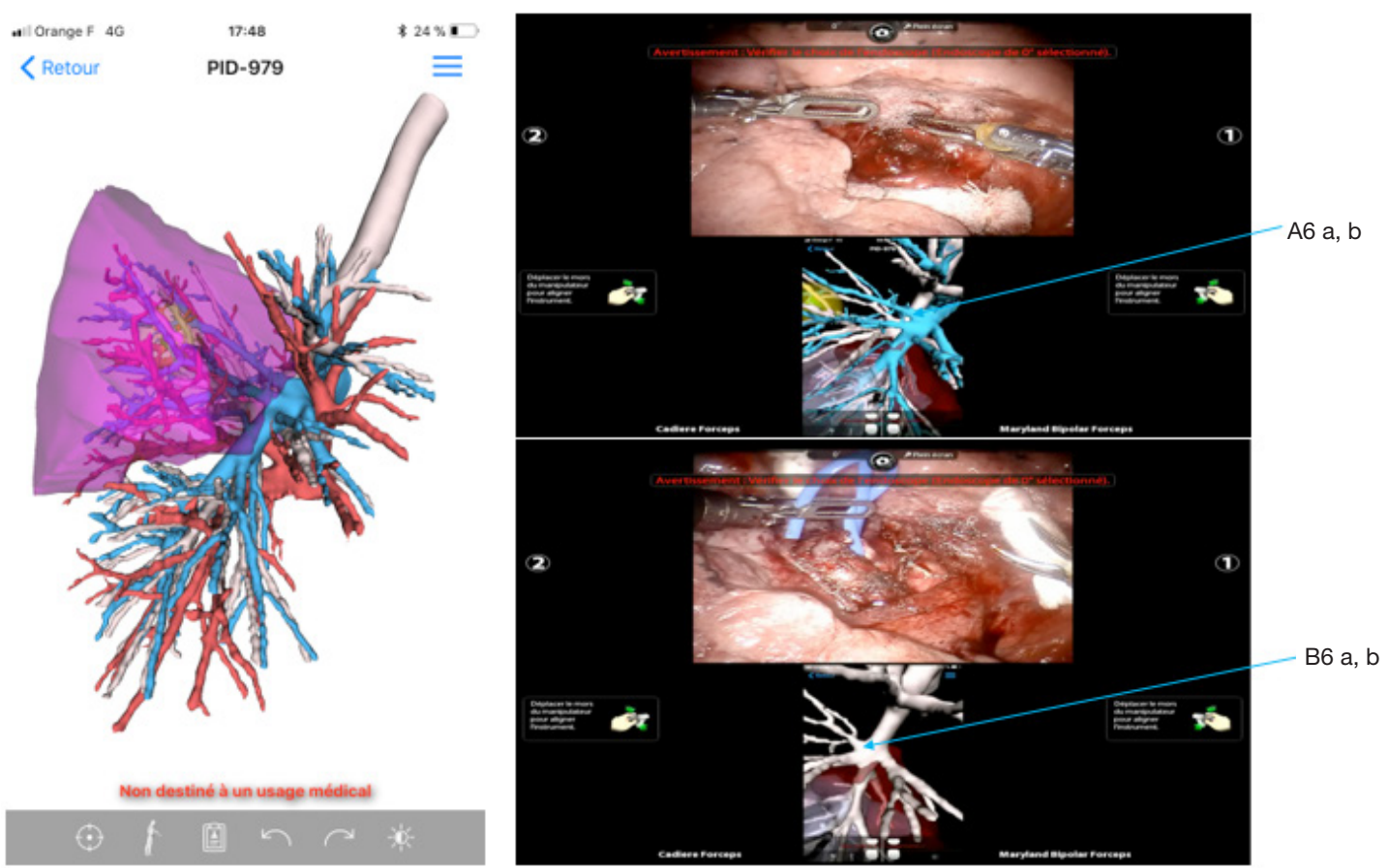

Figure 13 Right S6 segmentectomy with rare anatomical variation: 2 arteries and 2 segmental bronchus.

Patient ${ }^{\mathrm{TM}}$ and it was time consuming for the radiologist and the surgeon. The complex workflow involved in $3 \mathrm{D}$ reconstruction is the main drawback to its routine use in surgery. We have successfully simplified the workflow by associating Therapixel ${ }^{\mathrm{TM}}$ (Valbone, France) and Visible Patient ${ }^{\mathrm{TM}}$ (Strasbourg, France).

\section{Future developments}

This integrated system using a $3 \mathrm{D}$ model with preoperative marking if needed could also progress with fluorescence. The next step will be the integration of the NIR-ICG Fluorescence, Fire Fly System (Intuitive Inc., Sunnyvale, USA) in $2018(16,17)$. This integration will facilitate the localization of the intersegmental plan. After selective control of the segmental artery on the 3D model, the use of fluorescence will give us the perfect plan for the segmentectomy. This multimodal imaging system has been recently described to evaluate biliary anatomical variations and to secure the cholecystectomy. These visualization techniques could be a complement to reduce the likelihood of biliary injuries and in our study pulmonary injury (18). This system has been described as gall bladder navigation. By analogy we could describe our system as precision multimodal surgical navigation of the lung.
Another concept using a new fluorescent marker, folate receptor targeted near-infrared contrast agent (OTL38) can be used during robotic procedures and be added to the multi-modal system. This is known as intraoperative molecular imaging (IMI) (19). This new concept could replace pleural dye marking to assess the intersegmental plan for lesions close to two segments.

Our system is therefore adaptable and allows the addition of different technologies. This is the first step of imageassisted surgery. The near future will probably incorporate these technologies in one: robot and $3 \mathrm{D}$ reconstruction done in real time with augmented reality $(20,21)$. The field of interaction between the operator and virtual reality is rapidly evolving. The next step in the image overlay process is the integration of the $3 \mathrm{D}$ reconstructed structure directly in the stereoscopic view of the operating field offered by the robot without any input from the operator. A true augmented reality experience will soon be real and has already been successfully applied in other surgical fields (adrenalectomy, prostatectomy, hepatic surgery, neurosurgery, gynecology) (22).

Using currently available technology and with the support of the Visible Patient ${ }^{\mathrm{TM}}$ team, we managed to implement the use of computer-assisted surgery in our daily practice. The highly optimized model has a small size of 
only 15 to $25 \mathrm{MB}$, yet the representation of the anatomical accuracy of the bronchovascular elements, delimitation of each segment and lesion location are optimal.

\section{Limitations}

Ours is a composite system supported by three different companies: Therapixel ${ }^{\mathrm{TM}}$ for anonymization, DICOM transfer and screen support to view the $3 \mathrm{D}$ reconstruction, and Visible Patient ${ }^{\mathrm{TM}}$ which builds the $3 \mathrm{D}$ model and provides the software for use on a tablet or a phone. In addition, there is the robotic platform (Intuitive Inc ${ }^{\mathrm{TM}}$ ) which integrates the reconstruction in the operative field.

Associated with this relative complexity is the cost, which is a major obstacle to the development of this technology. The average cost for $3 \mathrm{D}$ segmentation is around 600 euros. It is likely that routine use of a $3 \mathrm{D}$ model will become the gold standard for minimally invasive pulmonary segmentectomy; hence, we need to work on lowering the cost and improving funding for this new surgical environment.

In our experience, the use of a multimodal imaging system combined with robotic surgery could improve the general performance of segmentectomy. However, after this descriptive study we have to prove the efficacy of this concept, which will be the next step in our work.

\section{Acknowledgements}

The authors are grateful to Nikki Sabourin-Gibbs, Rouen University Hospital, for her help in editing the manuscript. We also thank Visible Patient and Therapixel Compagnies for their great availability.

\section{Footnote}

Conflicts of Interest: Jean Marc Baste declares lecturing and proctoring for Intuitive Surgical. The other authors have no conflicts of interest to declare.

\section{References}

1. Landreneau RJ, D'Amico TA, Schuchert MJ, et al. Segmentectomy and lung cancer: why, when, how, and how good? Semin Thorac Cardiovasc Surg 2017;29:119-28.

2. Nagashima T, Shimizu K, Ohtaki Y, et al. Analysis of variation in bronchovascular pattern of the right middle and lower lobes of the lung using three-dimensional CT angiography and bronchography. Gen Thorac Cardiovasc Surg 2017;65:343-9.

3. Gossot D, Lutz J, Grigoroiu M, et al. Thoracoscopic anatomic segmentectomies for lung cancer: technical aspects. J Vis Surg 2016;2:171.

4. Pardolesi A, Park B, Petrella F, et al. Robotic anatomic segmentectomy of the lung: technical aspects and initial results. Ann Thorac Surg 2012;94:929-34.

5. Rinieri P, Peillon C, Salaün M, et al. Perioperativeoutcomes of video- and robot-assisted segmentectomies. Asian Cardiovasc Thorac Ann 2016;24:145-51.

6. Le Moal J, Peillon C, Dacher JN, et al. Three-dimensional computed tomography reconstruction for operative planning in robotic segmentectomy: a pilot study. J Thorac Dis 2018;10:196-201.

7. Lachkar S, Baste JM, Guisier F, et al. Pleural dye marking using radial endobronchial ultrasound and virtual bronchoscopy before sublobar pulmonary resection for small peripheral nodules. European Respiratory Journal 2017;50: PA3779.

8. Yang Q, Xie B, Hu M, et al. Thoracoscopic anatomic pulmonary segmentectomy: a 3-dimensional guided imaging system for lung operations. Interact Cardiovasc Thorac Surg 2016;23:183-9.

9. Baste JM, Soldea V, Lachkar S, et al. Visualization of the 3D model and exploration on the fluid console. Asvide 2018;5:406. Available online: http://www.asvide.com/ article/view/24363

10. Baste JM, Soldea V, Lachkar S, et al. Pleural dye marking before right $\mathrm{S} 1$ segmentectomy for better margin resection. Asvide 2018;5:407. Available online: http://www. asvide.com/article/view/24364

11. Baste JM, Soldea V, Lachkar S, et al. Left S6 with capture of the robotic console and 3D model. Asvide 2018;5:408. Available online: http://www.asvide.com/article/ view/24365

12. Baste JM, Soldea V, Lachkar S, et al. Double segmentectomies right S2 + S6 with capture of the robotic console and 3D model. Asvide 2018;5:409. Available online: http://www.asvide.com/article/view/24366

13. Baste JM, Soldea V, Lachkar S, et al. Left S2 segmentectomy using the per-operative $3 \mathrm{D}$ reconstruction. Asvide 2018;5:410. Available online: http://www.asvide. com/article/view/24367

14. Volonté F, Buchs NC, Pugin F, et al. Augmented reality to the rescue of the minimally invasive surgeon. The usefulness of the interposition of stereoscopic images in the Da Vinci ${ }^{\mathrm{TM}}$ robotic console. Int J Med Robot 
2013;9:e34-8.

15. Volonté F, Pugin F, Bucher P, et al. Augmented reality and image overlay navigation with OsiriX in laparoscopic and robotic surgery: not only a matter of fashion. J Hepatobiliary Pancreat Sci 2011;18:506-9.

16. Firefly fluorescence imaging. Available online: http:// www.roboticsurgery.com.au/product/firefly-fluorescenceimaging/

17. Zhou J, Yang F, Jiang G, et al. Applications of indocyanine green based near-infrared fluorescence imaging in thoracic surgery. J Thorac Dis 2016;8:S738-43.

18. Diana M, Soler L, Agnus V, et al. Prospective evaluation of precision multimodal gallbladder surgery navigation: virtual reality, near-infrared fluorescence, and X-ray-based intraoperative cholangiography. Ann Surg 2017:266:890-7.
19. Predina JD, Newton $\mathrm{AD}$, Keating J, et al. Intraoperative molecular imaging combined with positron emission tomography improves surgical management of peripheral malignant pulmonary nodules. Ann Surg 2017;266:479-88.

20. Soler L, Nicolau S, Pessaux P, et al. Real-time 3D image reconstruction guidance in liver resection surgery. Hepatobiliary Surg Nutr 2014;3:73-81.

21. Bourdel N, Collins T, Pizarro D, et al. Use of augmented reality in laparoscopic gynecology to visualize myomas. Fertil Steril 2017;107:737-9.

22. Khor WS, Baker B, Amin K, et al. Augmented and virtual reality in surgery-the digital surgical environment: applications, limitations and legal pitfalls. Ann Transl Med 2016;4:454.
Cite this article as: Baste JM, Soldea V, Lachkar S, Rinieri P, Sarsam M, Bottet B, Peillon C. Development of a precision multimodal surgical navigation system for lung robotic segmentectomy. J Thorac Dis 2018;10(Suppl 10):S1195-S1204. doi: $10.21037 /$ jtd.2018.01.32 